\title{
Composite Mantle Cell Lymphoma and "Gray Zone" Lymphoma in Two Different Anatomical Locations
}

\author{
Angeliki Cheva ${ }^{a}$, Triantafyllia Koletsa ${ }^{\mathrm{b}, \mathrm{c}}$, Leonidas Sakkas ${ }^{\mathrm{a}}$, Ioannis Kostopoulos ${ }^{\mathrm{b}}$
}

\begin{abstract}
Mantle cell lymphoma has been rarely found in composite form together with Hodgkin or non-Hodgkin lymphomas. A 69-yearold male patient presented with lumbal pain, cervical and axillary lymphadenopathy. Magnetic resonance imaging showed a tumor on T3/T4 extending to epidural region. Neurosurgical tumor excision was performed and the specimen along with a left axillary lymph node was sent for histologic examination, which showed infiltration of both specimens with neoplastic population, having morphologic and immunophenotypic characteristics of mantle cell lymphoma. Larger cells with morphological and immunohistochemical features of Hodgkin and Reed-Sternberg cells were also apparent, distributed diffusely among neoplastic mantle cells. Polymerase chain reaction analysis revealed $I G H$ monoclonality. On fluorescent in situ hybridization investigation, translocation $\mathrm{t}(11 ; 14)$ was detected. The interesting of this case rests in the presence of double neoplastic population in two different anatomical locations. The question raising in similar cases is whether this coexistence is coincidental or it is due to a clonal relationship. B-cell lymphoma, unclassifiable, with features intermediate between diffuse large B-cell lymphoma and classical Hodgkin lymphoma can be found in any site.
\end{abstract}

Keywords: Mantle cell lymphoma; Diffuse large B-cell lymphoma; Hodgkin lymphoma; Gray zone lymphoma; Composite lymphoma; FISH; EBER

\section{Introduction}

Mantle cell lymphoma (MCL), a pre-germinal center (GC)

\footnotetext{
Manuscript accepted for publication March 3, 2014

a Department of Pathology, General Hospital "George Papanikolaou", Thessaloniki, Greece

${ }^{\mathrm{b}}$ Department of Pathology, Medical School, Aristotle University, Thesssloniki, Greece

${ }^{\mathrm{c}}$ Corresponding author: Triantafyllia Koletsa, Pathology Department, Medical School, Aristotle University of Thessaloniki, University Campus, 54124 Thessaloniki, Greece. Email: koletsa@med.auth.gr
}

doi: http://dx.doi.org/10.14740/jmc1718w lymphoma, has been described in composite form together with GC-derived lymphomas, such as follicular or Hodgkin lymphoma (HL) $[1,2]$, or with post-GC neoplasms, such as small lymphocytic lymphoma and plasmacytoma $[3,4]$. MCL and diffuse large B-cell lymphoma (DLBCL) are an uncommon event with only two reported cases in the literature [5]. Herein, a unique case of composite mantle cell and "gray zone" lymphoma with morphologic and immunophenotypic features between DLBCL and HL is described.

\section{Case Report}

A 69-year-old male patient presented with lumbal pain. Clinical examination revealed enlarged left cervical and axillary lymph nodes. Computed tomography scan of the chest and abdomen showed enlarged para-aortical lymph nodes, as well. No masses or organomegaly was found. Magnetic resonance imaging revealed a tumor on $\mathrm{T} 3 / \mathrm{T} 4$, extending to epidural and pressing on the spinal cord (Fig. 1a, b). Routine laboratory tests were within normal ranges. Chest X-ray was unremarkable. Neurosurgical tumor excision was performed and the specimen along with a left axillary lymph node was sent for histologic examination.

Histologic examination of epidural tumor showed neoplastic population consisted of medium-sized neoplastic lymphoid cells with scant cytoplasm and round or ovoid nuclei with indistinct nucleoli. A moderate number of mitoses were observed. Among these neoplastic cells, large cells were diffusely observed, many of which had centroblastic or immunoblastic features, while there were few with characteristics of Hodgkin and Reed-Sternberg cells (HRS). In some areas fine, incomplete fibrous septa were also apparent. On immunohistochemical analysis, the medium-sized lymphoid population showed the following immunophenotype: CD45+,CD20+, CD79a+, PAX5+, BCL2+, cyclinD1+, Oct2+, BOB.1++/-, CD5-, CD3-, CD23-, BCL6-, CD10-, MUM1-, CD30-, CD15-, EMA-, ALK-1- and LMP1-. On the other hand, large and RS cells were positive to CD20, PAX5, CD30, CD15, BCL2, Oct2 and MUM1 immunostains and only focally to CD79a and BOB1, whereas all the other above mentioned markers were negative. 

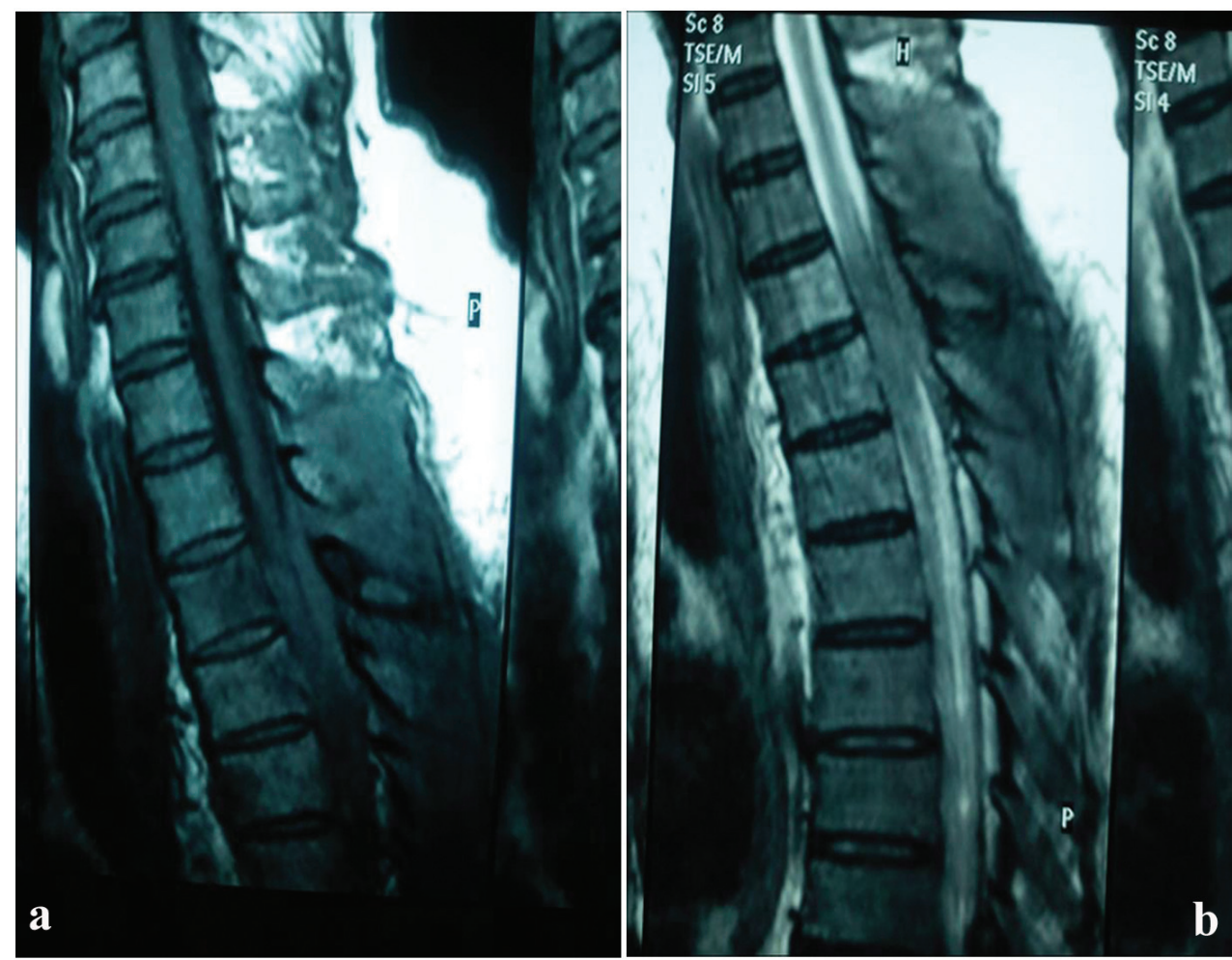

Figure 1. Sagittal post-contrast T1-weighted image of the thoracic spine reveals a lesion in the posterior (increased signal intensity) epidural region compressing the cord (a). Sagittal T2-weighted image of the thoracic spine at the same level depicts the same lesion in the posterior epidural region with low signal intensity (b).

Histologic examination of lymph node showed total effacement of the architecture because of the above described double neoplastic population (Fig. 2a). In addition, large and HRS cells were noticeably more and arranged closely, having a syncytial appearance. It is of note that no inflammatory cells were apparent and the HRS cells were surrounded by the medium-sized neoplastic population (Fig. 2b). The immunophenotype was identical to that observed in epidural tumor (Fig. 2b, d). Moreover, in situ hybridization with Epstein-Barr encoding region was performed, revealing negativity of both MCL and RS cells for Epstein-Barr virus.

Molecular analysis by polymerase chain reaction revealed $I G H$ monoclonality. Fluorescent in situ hybridization (FISH) analysis showed $\mathrm{t}(11 ; 14)$ in neoplastic mantle cells (Fig. 2e). It was not possible to proceed to microdissection in order to confirm if the double neoplastic population had identical $I G H$ rearrangements or to be sure that the large lymphoma cells were investigated for translocation $\mathrm{t}(11 ; 14)$. Bone marrow biopsy was negative for lymphoma infiltration.

\section{Discussion}

Composite lymphoma is the term used to describe a single anatomic site involved by two distinct types of lymphoma.
Globally, the question raising in composite lymphomas is whether this coexistence is coincidental or it is due to clonal relation. In recent years, laser microdissection and molecular analysis of composite lymphomas have shown either unrelated clonal rearrangements [6] or a common clone-specific rearrangement, suggesting the unusual evolution of a single malignant clone to two different lymphomas $[1,2]$. In this reported case, immunohistochemical characteristics of the neoplastic cells do not allow us to hypothesize a relationship of the two neoplastic populations. Even cyclinD1 immunostain was negative to large cells. The fact that the double population was found in two different locations may indicate that there is an association between the two neoplasms. On the other hand, microenvironment may be responsible for the coexistence of two clonally independent neoplastic populations.

In this case CD5 antigen was not expressed in neoplastic cells and the diagnosis of MCL was confirmed by cyclinD1 positivity and FISH analysis, showing translocation $\mathrm{t}(11 ; 14)$. The main diagnostic problem in similar cases is the identification of the large cells. They could not represent large pleiomorphic mantle cells, since cyclinD1 was negative. The immunophenotypic features resembled those of classical Hodgkin lymphoma (cHL). Coexpression of CD30 and CD15 antigens argues for $\mathrm{cHL}$, and it has been also de- 

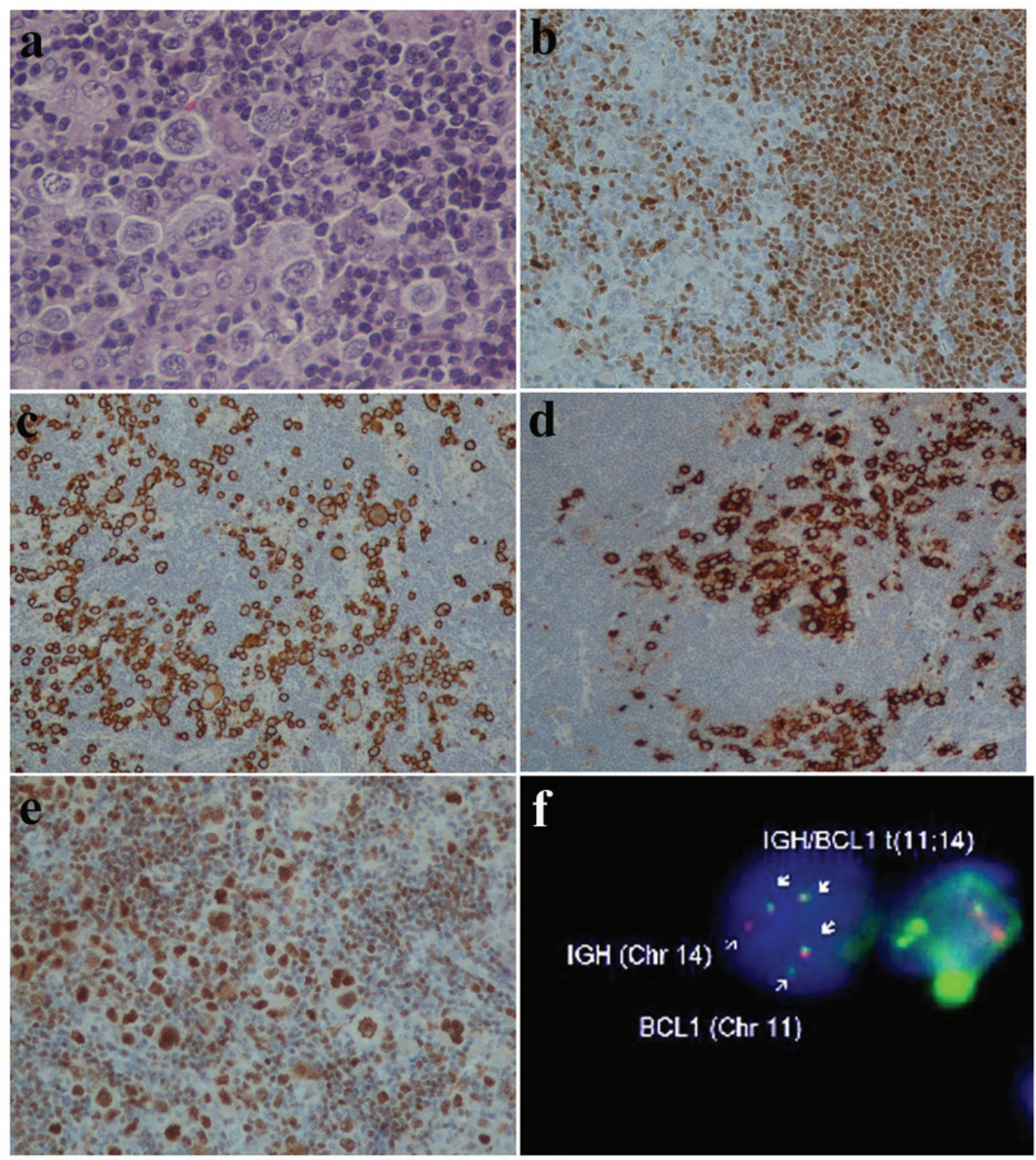

BCL1 (Chr 11)

Figure 2. Neoplastic mantle cells and RS-like cells (a). Mantle cells positive to cyclinD1 (b) and RS cells immunoreactive to CD30 (c) and CD15 (d). Oct2 strong positivity of MCL and HRS cells (e). FISH method with IGH (red) and BCL1 (or CCND1) (green) gene probes revealing IGH/CCND1 gene fusion (arrows) (f). (a: HE, × 400; b, e: IHC, × 200; c, d: IHC, × 100).

scribed in gray zone lymphomas $[7,8]$. The absence of the appropriate inflammatory background of cHL may be due to the presence of neoplastic mantle cells. On the other hand, immunoreactivity to CD45, CD79a, BOB.1 and Oct-2 antigens favors DLBCL diagnosis. Hence, this lymphoma is a B-lineage lymphoma with overlapping morphological and immunophenotypic features between cHL and DLBCL.

In 2008 World Health Organization classification of a new category of lymphoma was introduced under the term B-cell lymphoma, unclassifiable, with features intermediate between DLBCL and cHL for those cases with overlapping features between $\mathrm{cHL}$ and DLBCL, especially primary mediastinal large B-cell lymphoma [9]. Although this entity concerns mainly the mediastinum, it can also include lymphomas occurring in other anatomical sites [7-9]. The immunophenotype is typically borderline between that of cHL and DLBCL with neoplastic cells expressing CD45, CD20, Oct 2, Bob.1, CD30 and often CD15 [9, 10].

Conclusively, any lymphoma can be found simultaneously with another lymphoma type in a composite form. Composite lymphomas can occur in every anatomical location. Microenvironmental factors may play an important role in finding the same composite lymphoma in different anatomical sites. Borderline immunophenotype, characterized 
by positivity to CD45, CD20, Oct 2, Bob.1, CD 30 and CD 15 antigens, helps to diagnose unclassifiable B-cell lymphoma with features intermediate between DLBCL and cHL.

\section{Conflict of Interest}

The authors declare no conflict of interest.

\section{References}

1. Wang S, Tzankov A, Xu-Monette ZY, Hoeller S, Wang SA, Richards KL, Zhang S, et al. Clonally related composite follicular lymphoma and mantle cell lymphoma with clinicopathologic features and biological implications. Hum Pathol. 2013;44(12):2658-2667.

2. Tinguely M, Rosenquist R, Sundstrom C, Amini RM, Kuppers R, Hansmann ML, Brauninger A. Analysis of a clonally related mantle cell and Hodgkin lymphoma indicates Epstein-Barr virus infection of a Hodgkin/ReedSternberg cell precursor in a germinal center. Am J Surg Pathol. 2003;27(11):1483-1488.

3. Cachia AR, Diss TC, Isaacson PG. Composite mantle-cell lymphoma and plasmacytoma. Hum Pathol. 1997;28(11):1291-1295.

4. Hoeller S, Zhou Y, Kanagal-Shamanna R, Xu-Monette ZY, Hoehn D, Bihl M, Swerdlow SH, et al. Composite mantle cell lymphoma and chronic lymphocytic leukemia/small lymphocytic lymphoma: a clinicopathologic and molecular study. Hum Pathol. 2013;44(1):110-121.

5. Ho AK, Teman CJ, Smith GP, Nightingale DR, Miles RR. Composite mantle cell and diffuse large B-cell lymphoma: report of two cases. Int J Surg Pathol. 2011;19(5):643-648.

6. Caleo A, Sanchez-Aguilera A, Rodriguez S, Dotor AM, Beltran L, de Larrinoa AF, Menarguez FJ, et al. Composite Hodgkin lymphoma and mantle cell lymphoma: two clonally unrelated tumors. Am J Surg Pathol. 2003;27(12):1577-1580.

7. Garcia JF, Mollejo M, Fraga M, Forteza J, Muniesa JA, Perez-Guillermo M, Perez-Seoane C, et al. Large B-cell lymphoma with Hodgkin's features. Histopathology. 2005;47(1):101-110.

8. Wang E, Foo WC, Huang Q. A sinusoidal large cell lymphoma with expression of CD30, CD15, and multiple Bcell antigens: a classical Hodgkin lymphoma with sinusoidal infiltrating pattern or a sinusoidal CD30 positive large B-cell lymphoma with CD15? Leuk Lymphoma. 2010;51(6):1148-1151.

9. Jaffe ES, Stein H, Swerdlow SH, Campo E, Pileri SA, Harris NL. B-cell lymphoma, unclassifiable, with features intermediate between diffuse large B-cell lymphoma and classical Hodgkin lymphoma. In: Swerdlow SH, Campo E, Harris NL, et al. (Eds). WHO classification of tumours of haematopoietic and lymphoid tissues. Lyon: IARC Press; 2008. pp. 267-268.

10. Harris NL. Shades of gray between large B-cell lymphomas and Hodgkin lymphomas: differential diagnosis and biological implications. Mod Pathol. 2013;26 (Suppl 1):S57-70. 